\title{
Exploring Approaches to Teaching Bilingual Education in Countries where English is a First Language: A study in East London
}

\author{
Mohammad Rasel Howlader
}

\begin{abstract}
The aim of the study is to recognize literacy practices of the ethnic minorities and find suitable teaching approaches in a multilingual context in London. The main research question is: How do the ethnic minorities in London want their heritage languages to be recognized in their mainstream schools? Primary research has been carried out through questionnaires and structured interviews and secondary research has been carried out through analyzing published books and articles. In breaking the silence of the bilingual children, it has been suggested that a post communicative approach would be convenient to teach ethnic minority languages and activities like pair work, group work, role play, brainstorming, multiple choice and filling in the gaps would be suitable in bilingual learning. Results show that bilingual learning can be enhanced through parental support, social media, storytelling by grandparents and the inclusion of a bilingual syllabus in the National Curriculum in England.
\end{abstract}

Keywords: bilingual learning, breaking bilingual silence, recognize literacy practices, post communicative approach

\section{Introduction}

The aim of this paper is to find different ways to recognize literacy practices of the ethnic minorities and teach their heritage languages in East London. The main focus has been given to British born Asian children who are studying in mainstream schools. I have chosen this research topic to break the silence of the ethnic minority children in London and to show their multilingual identity without any hesitation in a multilingual society. The main research question is: How do the ethnic minorities in London want their heritage languages to be recognized in their mainstream schools?

The study has been carried out using a mixed method. I have used qualitative method such as structured interviews, observations and quantitative method such as questionnaire to collect primary data and to find out different ways to recognize and teach heritage languages to multilingual children in London. The current research is not focusing on a large scale investigation but on a small number of 
students where closer attention has been given to collect realistic data from the respondents of this study.

\section{Theoretical Framework}

English has become a powerful global language since its colonial power with language influence in America, Australia, India and many other countries of the world (Harmer, 2003, pp. 14-16). English language teaching is sometimes viewed "as a form of cultural or linguistic imperialism" (Harmer, 2003, p. 16, Phillipson, 1992, Pennycook, 1994). English is used to promote military, cultural and economic hegemony (Said, 1978, Harmer, 2003, p. 16). Harmer (2003) believes that nonnative speakers of English are victims of linguistic and cultural imperialism or linguistic capital (Bourdieu, 1991). Harmer (2003) is concerned with language loss as "language death is frightening ......and its loss takes with culture and customs"(p. 16). Some countries like Nigeria have taken initiatives to keep other languages alive (Harmer, 2003). He also shows that there are more than one languages being operated in Nigeria. English is the key language and other languages are surviving depending on the situation. Extraordinary Nigerian writers like Achebe, Soyinka and Ngugu do not write in English (Harmer, 2003, p. 16). Multilingualism is acceptable in Nigeria.

London, a multilingual city, has many complementary schools. For example, there are $90 \%$ Bengali speaking people in the Tower Hamlets area. Bengali is spoken in the wider society (Kenner \& Ruby, 2012). Unfortunately, Bengali is not spoken overtly in the main stream schools. Most of the school children are keen to show their identity in the dominant English language. Some private initiatives have been taken to legalize the ethnic minority languages like Bengali and Urdu. Apart from learning Bengali, Muslim students learn the Arabic language to perform their religious activities (Kenner \& Ruby, 2012).

There is no denying the fact that English is the "linguistic capital" (Bourdieu, 1991) and highly accepted "legitimate language" (Kenner \& Ruby, 2012) in the school curriculum in the UK. However, there are strong grounds to argue that multilingual learning would not affect or belittle this "legitimate language" (Kenner and Ruby, 2012) in wider society. In fact, the prestige and honor of the influential language will be uplifted in the sense that it is generous enough to provide with "consent" (Said, 1978) for creating rooms for multilingual learning. The United Kingdom is a multilingual and multicultural country (Kenner \& Ruby, 2012). However, children's heritage languages are at risk of extinction. It is widely accepted that multilingual learning can enhance second language learning (Kenner and Ruby, 2012, Cummins, 1984, Baker, 2011).

\section{L1 can help to improve $L 2$}

There are some common rules of grammar in all languages. For example, in Bengali the subject comes at the beginning of a sentence and the adjective comes before noun. In Bengali sentence, "Peter hoy akjon valo manus", Valo=adjective (good), manus=noun (Noun). The same pattern can be observed in English. For example, in 
the sentence "Peter is a good man", Peter (Sub)+ is (be verb)+ a (article) + good (adjective)+ man (noun). Their existing knowledge can help them to improve learning second languages. Anderson and Obied (2011) have positively underpinned socio-constructivism theory of Vygotsky, Bruner and Mercer (p.16). According to them new ideas and knowledge are built on existing knowledge within "the Zone of proximal development (ZPD)", more capable peer can help the less capable peer to develop language learning. Anderson and Obied (2011) believe "scaffolding strategies play an important role in mapping pathways and developing confidence" (P. 19). Therefore, heritage language is the strong basis to learn further languages.

\section{Scaffolding and teaching assistant}

The previous paragraph underpinned the ideas of ZPD and scaffolding. The idea of employing heritage language teaching assistants in mainstream schools should not be ignored as they could be ideal figures of more capable peers according to Vygotsky's ZPD and Bandura's scaffolding. Miller (2010) shows Vygotsky's understanding of the Tools of development. Adults can guide children about how to use different tools of the environment. Proper mediation between the child and the environment is worth considering. Tools develop different factors in the child's mind. The environment provides all sorts of facilities for the child. Through a proper association between the child and the society development happens. There are two types of tools which aid us in our mental functioning. Firstly, psychological tools which include language systems, counting systems, writing and works of art. These are used to control thought and behavior. In the classroom, a teacher can show the process of writing a paragraph. Children can observe their teacher and take initiatives to write their own. Secondly, technical tools are used to control nature these are pen, computer for example.

Vygotsky believes that language, the psychological tool, enables a child to depict the time variation including the past, present and future. In Vygotsky's words, "just as mole gives shape to a substance, words can shape an activity into a structure" (1978, pp. 28 in Miller 2010, p. 384). Central to Vygotsky's theory is the child's engagement in cultural activity with guidance from more skilled others-peers and adults alike. Therefore, the teacher and knowledgeable peer is a facilitator in children's learning (Vygotsky, 1978). Collaboration with adults and with other children plays a central role in the cognitive development of children. Therefore, working with the assumption that learning occurs primarily in social interaction, when learners interact collaboratively with more knowledgeable others while passing on to the child the cultural tools required in the specific society.

Ethnic minority children in the UK should not be pressurized to learn only the "legitimate language" (Kenner \& Ruby, 2012). It affects their cognitive development. Children in the main stream schools do not get the opportunity to express their multilingual identity. There should be suitable rooms for the bilingual children in the wider society. Policy makers in the curriculum should consider that children's heritage language can develop second language learning. All language 
learning starts from the same "engine" (Cummins, 1981a) or "Language Acquisition Device" (Chomsky, 2007). Children can develop their cognitive development nicely through acquisition of heritage language. Multilingual identity should be respected everywhere as the UK is a multilingual and multicultural country. Children should feel at ease with their heritage culture, language and identity. It will strengthen their sense of individual identity in this country.

Baker (2011) suggests that cognitive development advances when children become relatively balanced bilingual (p. 165). His research suggests that bilingual education including "immersion" and "heritage language bilingual education" (Baker, 2011, p. 165) result in performance enrichment in two languages and in general curriculum performances compared to monolingual education. The brain in human head has ample space for language skills. Baker (2011) emphasizes that language learning does not occur separately but there is "a common underlying proficiency" (Baker, 2011: 165) which is interactive. Therefore, ideas learnt in one language can be easily transferred to another language. In this instance, there is no separate feeling of an Urdu part of the brain or Russian part of the brain in terms of school lesson learning. Baker (2011) appreciates Cummins' (1981a) “Common Underlying Proficiency" model as its concept is easy to understand pictorial representation of two icebergs. Two icebergs are separate above surface of water. However, they have close link with each other through the surface. Underneath the surface two icebergs are completely fused and interactive. Similarly, two languages do not function separately. They function and operate through the same central processing system. Therefore, the central processing engine can operate more than two languages. It can easily result in multilingualism (Baker, 2011: 167).

A bilingual child's mind is ready to receive skills and metalinguistic knowledge through the interference of first and second language (Cummins, 1981). In relation to this, Cummins (1981) argues that this interference occurs due to the "common underlying proficiency" which is like two ice bergs floating on the same water level. His theory leads to acceptable circumstance that learning one language will have a positive impact on the additional language learning.

\section{Research methodology}

I carried out the study using a mixed method. I used qualitative method such as structured interviews and observations and quantitative method such as questionnaires to investigate the current situation of the ethnic minority children in London and find out suitable approaches to develop multilingualism. Throughout the study I maintained confidentiality while collecting personal data on bilingual issues.

Thirty informants took part from different mainstream schools in East London. Most of the respondents belong to the British born Asian community in London. Informed consent via mobile phone has been sought for the questionnaires and structured interviews. Before sending out questionnaires, I made sure that all the respondents understood the research questions. Piloting of some questionnaires made me clear that the respondents understood questionnaires. Apart from this, I 
collected recorded consent from four interviewees. I maintained confidentiality in all cases. For example, all the respondents were assured through written statements that their real name will not be used in the study. I have consulted British Educational Research Association (BERA) website to ensure that all ethical issues have been maintained while conducting this research study.

\section{Results and findings}

I carried out the study with thirty four respondents. Thirty respondents participated in the questionnaire and four respondents took part in the interview sessions. Most of the respondents are British-born Asian children and a minority of the respondents are British born Polish, Somali, Arabian and Jamaican children. Most of the children's ages range from nine to thirteen. The majority of them are studying in mainstream school and some of them are studying in Muslim school. I prepared suitable questionnaires and structured interviews for these children so that they could understand the research clearly. Most of the respondents came forward to share their bilingual identity.

\section{Questionnaire analysis}

\section{Multilingual identity}

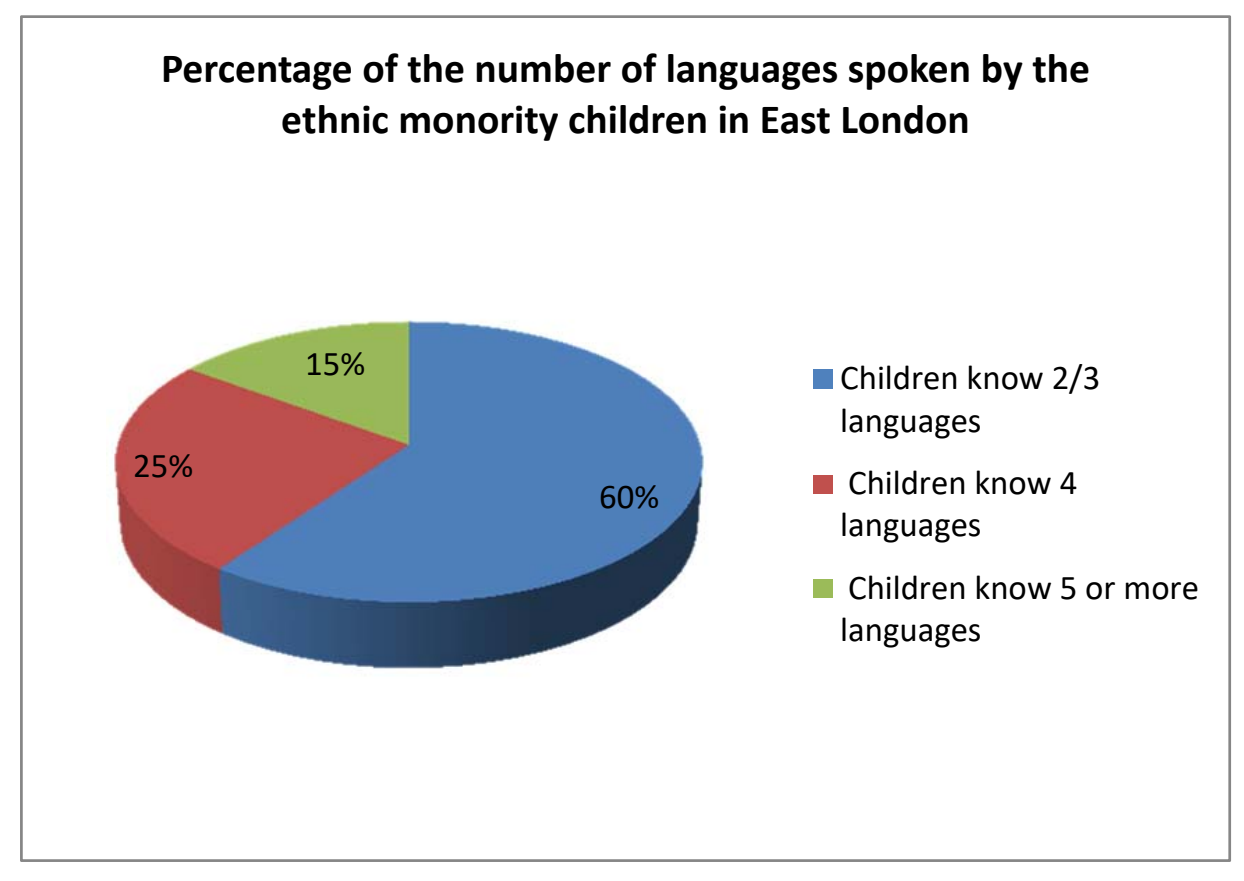

Pie chart no---01

The study has identified remarkable increase of bilingual speakers in East London where ethnic minorities knowing two languages are more dominant. This study was 
carried out in East London area where most of the respondents are British born Bangladeshi, Indian and Pakistani.

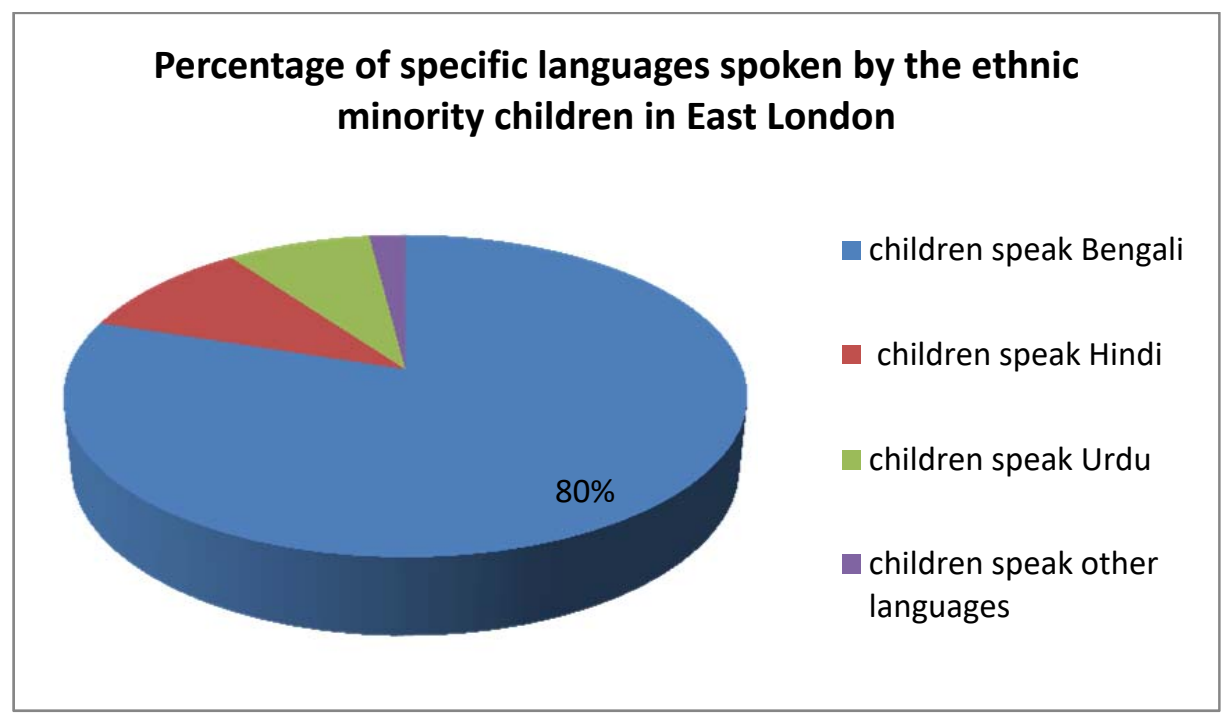

Pie chart no-02

There are more Bengali heritage speakers in East London noticeably in Tower Hamlets area. There are Urdu, Hindi and other speakers in small proportion as well.

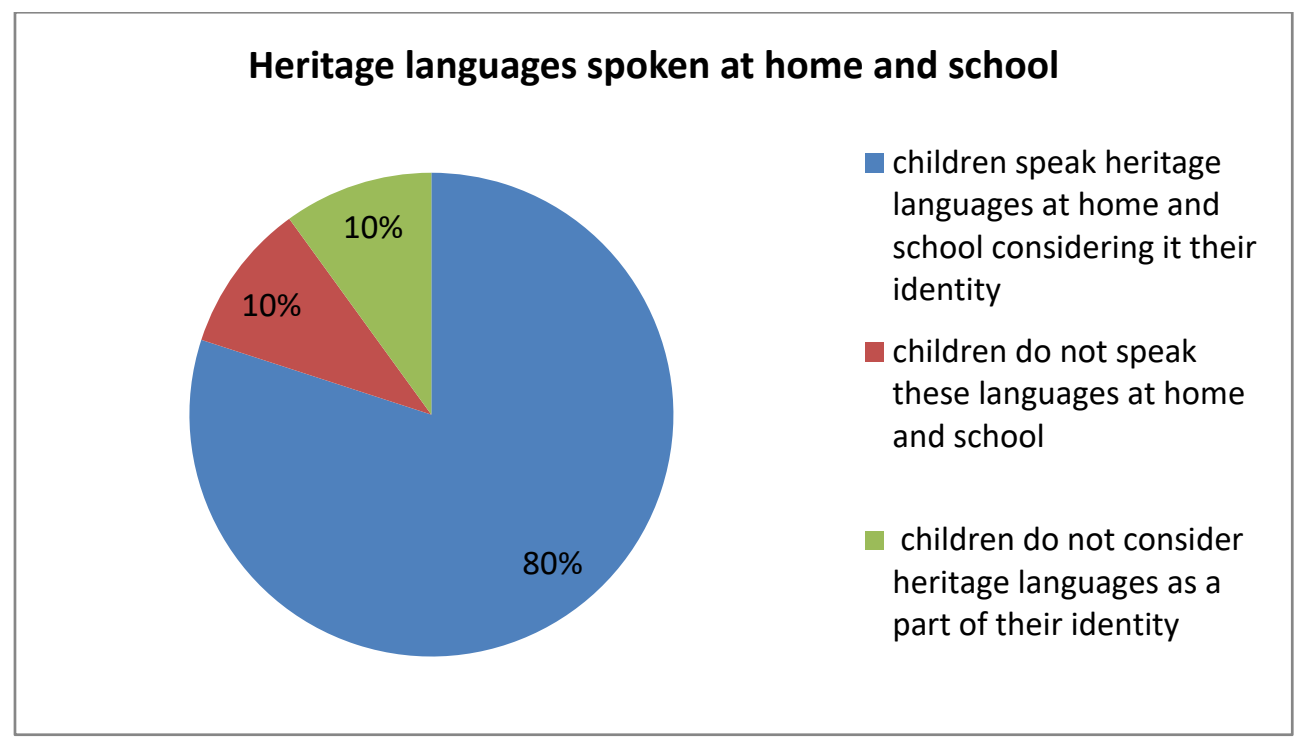

Pie chart no- 03

Most of the ethnic minorities in East London consider their heritage languages as part of their identities. They find possible ways to apply their heritage languages both at home and schools depending on situations. A small number of ethnic minorities are reluctant to use their heritage languages. Data analysis reveals that some mainstream schools in the UK have restricted heritage language use 
where "symbolic violence' could take place (Bourdieu, 1991). These children do not feel the urge to practice heritage languages at home. They are more used to the standard variety of English (Kenner \& Ruby, 2012).

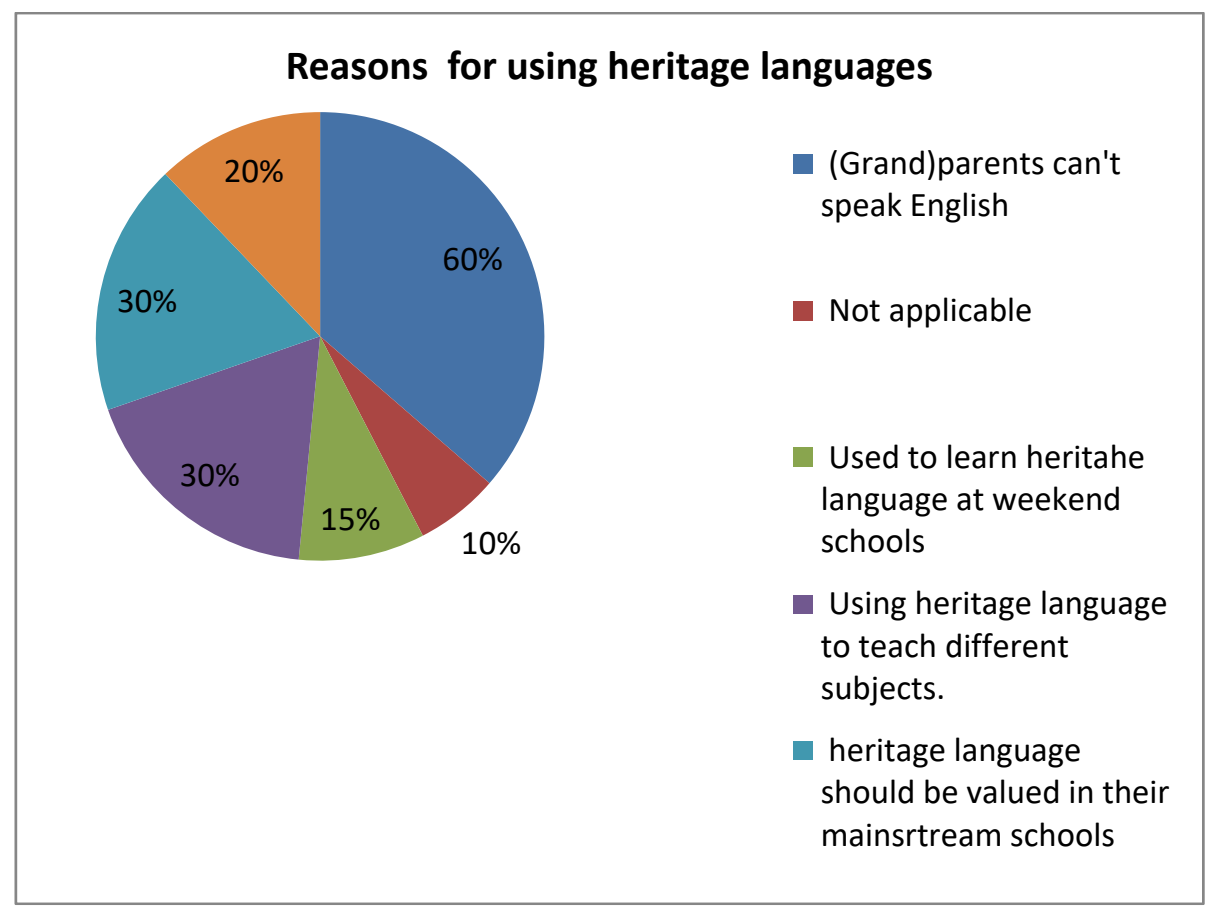

Pie chart no-04

Most of the children are second or third generation British born Asian children. Most of their parents or grandparents cannot speak English. But they allow their children to study in mainstream schools to secure a place in job market (Kenner and Ruby, 2012). Their children are bound to face the challenges in two ways. Firstly, they have to speak their heritage languages at home to communicate with their parents and grandparents. Secondly, they have to learn standard variety of English to show they are more British (Kenner and Ruby, 2012).

The graph shows that $30 \%$ children with the same cultural and linguistic background used to attend weekend schools to learn heritage languages. For example, in the Tower Hamlets area, there are many complementary schools. Most of the students are Bengali. They learn their heritage language to interact and have daily conversations with their parents and grandparents.

The graph shows that $30 \%$ children consider that their heritage languages should be valued in their mainstream schools. Sometimes, there can be a couple of children from the same cultural and linguistic background. They might feel it is convenient to share their experience of study in their heritage language. In this circumstance, other monolingual children should not mock the ethnic minority children. They feel that their school authority should take initiatives so that other students do not bully ethnic minority children. Such as, they think that their heritage languages can be used to teach other subjects religious studies or literature. They 
can interpret one object in two or more words. Their understanding of study will be enhanced more following inclusion of their heritage languages to teach other subjects (Kenner \& Ruby, 2012).

This pie chart also demonstrates that 20\% children are attending weekend schools to learn their heritage languages. They consider their heritage languages important in their lives. They feel that their parents or grandparents cannot speak proper English. They can express their cultural and linguistic experiences with their parents and grandparents. There are qualified teachers for heritage languages. Most of them have qualification from their home country (Kenner \& Ruby, 2012). Some of them have gained additional qualification in the UK like PGCE. They collect course materials from their home country and teach in weekend schools (Kenner \& Ruby, 2012). However, materials collected for teaching heritage languages are not sufficient. Materials produced in the UK for teaching heritage languages might enhance bilingual learning.

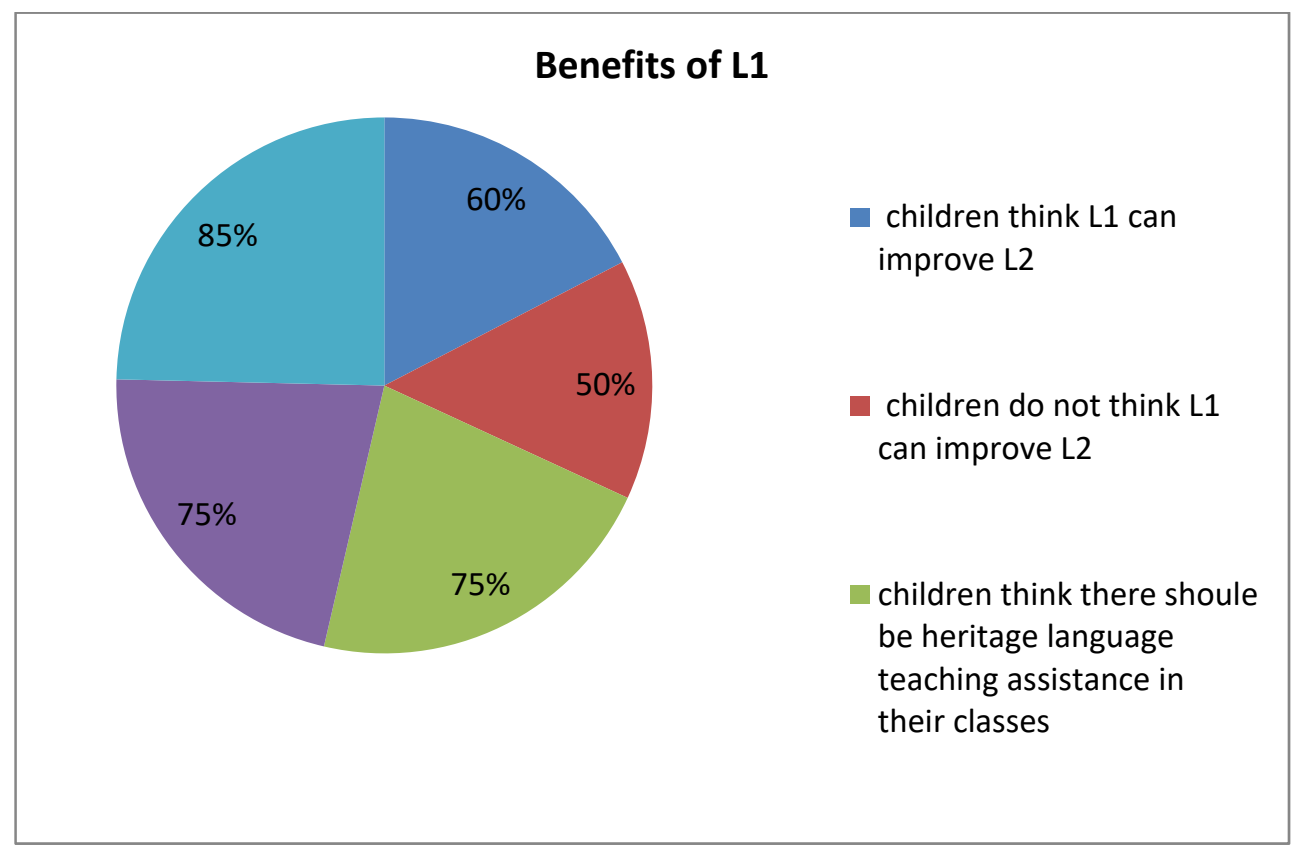

Pie chart no-5

Most of the children in this study believe that employing heritage language teaching assistants would contribute more to their cognitive development. Children use their heritage language to gain linguistic smartness, to translate other languages and to secure a job. For example, when they become adult, they can do interpreting job. Many visitors come to the UK every year for treatment or business purpose. They can earn a lot of money by working as interpreters. They do not want to lose their bilingual identity in the monolingual influential society. Bilingual identity can enhance their professional development in future as well. 


\section{Inclusion of heritage languages in curriculum}

This pie chart also shows that $75 \%$ children want their heritage languages as a part of national curriculum. Although British curriculum allowed some dominant minority languages like French and Spanish in the national curriculum, recent report on education suggests that the government of the UK will allow other languages to be a part of the national curriculum. Children feel that inclusion of their heritage languages in the national curriculum of the UK would make them more in their education.

$85 \%$ children do not show their bilingual identity because of the monolingual environment in mainstream schools. They are more interested to show their multilingual identity provided that they get suitable room to do so. Their monolingual silence (Kenner \& Ruby, 2012) can be broken by including their heritage language in the national curriculum of the UK and keeping teaching assistants in their respective heritage languages. However, I believe that it is not possible to keep heritage language teaching assistants for a very small number of ethnic minority children. It would probably be logical to keep heritage language teaching assistants for a good number of ethnic minority children.

Some children in the questionnaires (question no-14) have commented that they are learning the dominant English language for future professional development. Similarly, British children can try to learn Bengali language for cognitive development and getting job of interpreter in future. Therefore, language learning does not go in vain. If this reciprocal learning process continues, ethnic minority children can remove their bilingual shyness.

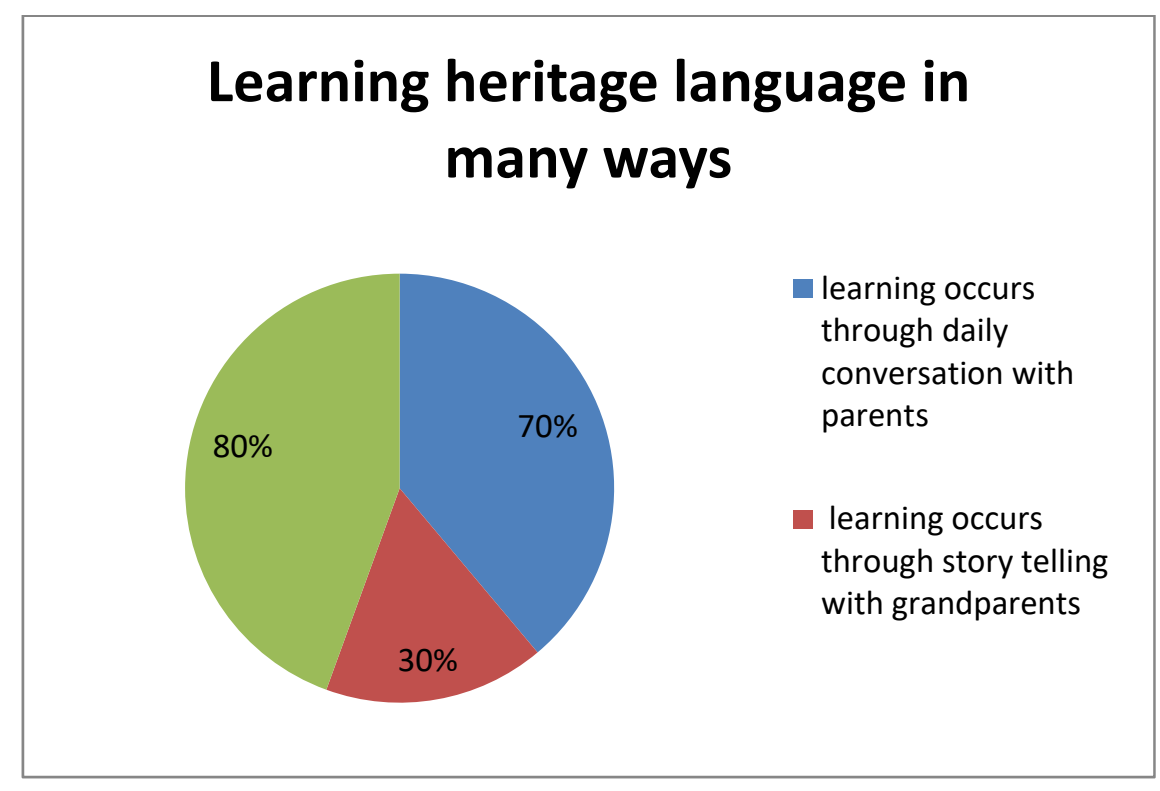

Pie chart no-06

This pie chart shows that $70 \%$ children learn their heritages languages through daily conversation with parents and 30\% learn by storytelling from 
grandparents. East London is a place where most of the Asian people live. Therefore, $80 \%$ children do not see any problem using their heritage languages in the place where they live.

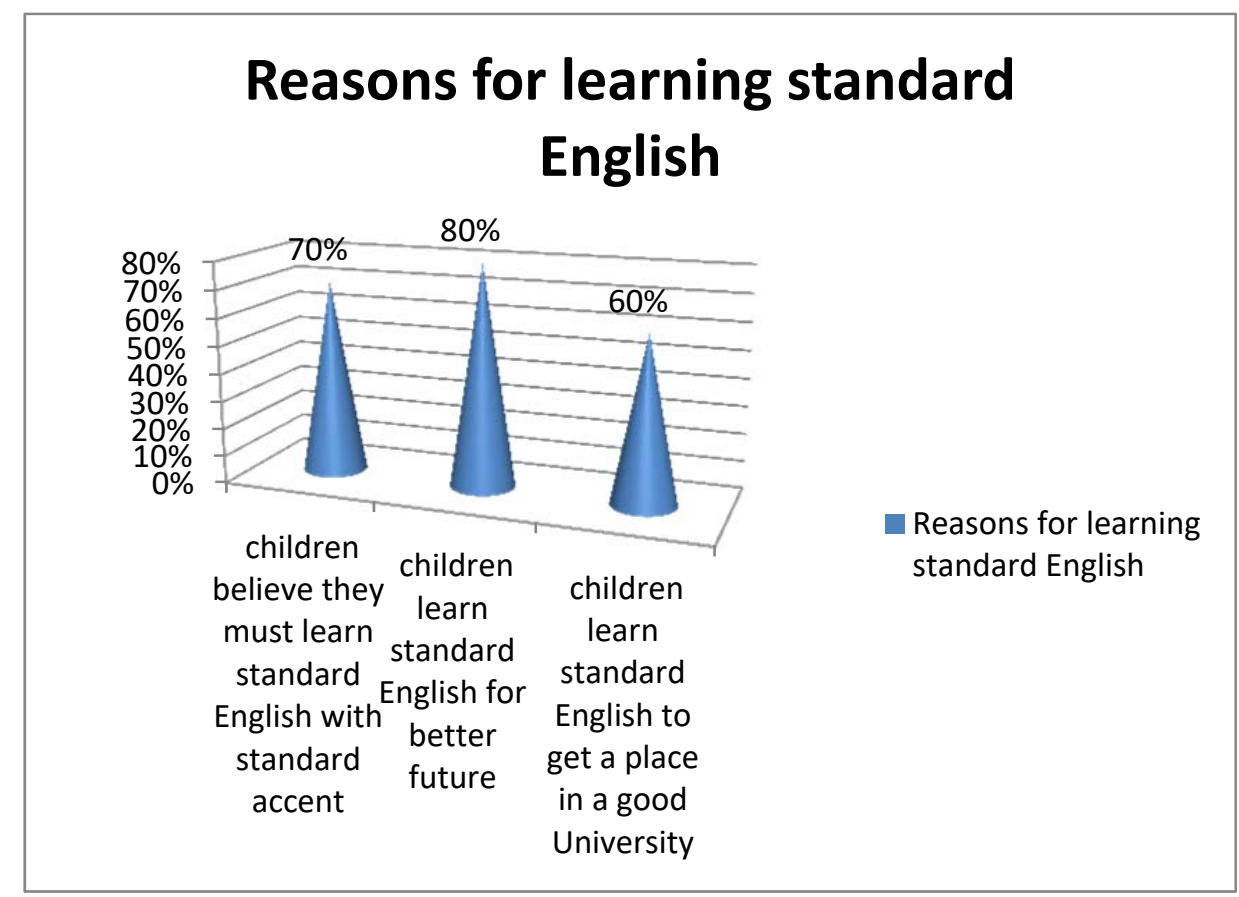

\section{Graph no-01}

More than 70\% ethnic minorities in East London acknowledge that they must learn Standard English with standard accent. They need Standard English for a better future. In this regard, the percentage stands at $80 \%$. On the other hand, $60 \%$ children learn Standard English to secure a place in a good University. They all are concerned about learning Standard English with Standard English accent. Mainstream schools are the only places for them to develop standard variety of English. Moreover, a good number of children feel that they can contribute more to the place where they live by learning their heritage languages. They can retain their respective culture and identity in their respective living place by improving their heritage languages.

\section{Structured Interview}

The study conducted four structured interviews from three primary schools in East London. Previous paragraphs discussed the need of Standard English for a better future and heritage languages for communicating and interpreting purposes. Most of the major dialogues of the respondents show the same concern. Major dialogues have been presented below without any alteration.

\section{Interview 1}

Interviewer: Rasel, Participant: Ibrahim Rasel: Are you interested in speaking more than one language? Which languages? Ibrahim: Yes, Sometimes, if it is easy, I will be able to learn quickly and learn more 
languages....

Rasel: Which languages?

Ibrahim: Urdu! And Arabic.....I wanna go to foreign places like Saudi Arabia and Middle East and Bangladesh..................

Rasel: Why Bengali?

Ibrahim: Bengali is my heritage language. My forefathers and father used to use Bengali language

Rasel: Why do you use more than one language?

Ibrahim: I wanna go to other country. I can't speak their language. I will be able to communicate with some many people.

Rasel: Which language do you usually speak at home and why?

Ibrahim: English...... s speak English. Because everyone knows a lot of English and feel comfortable with English.

Rasel: Why are you not comfortable with heritage language?

Ibrahim: Because, I don't feel comfortable, when I was a baby I was bought up in English, I wasn't brought up in Bengali, So I feel weird speaking Bengali. I know my mom and aunty speak English.

Rasel: Do you use your heritage language in the place where you live? When and in which situations?

Ibrahim: I do.....like I already said; my dad and my grandparents speak heritage language, Er... I speak heritage language when I go to Bangladesh.

Rasel: If you were given the opportunity, which language would you want to be included in the school curriculum apart from English?

Ibrahim: Arabic.......we believe life after death....... So If I learn Arabic, I will be able to answer questions in Arabic in the day of judgement....it is the language of the Holy Quran.

Rasel: What do you think students learn Standard English in school?

Ibrahim: Because, students should learn standard English...the whole world English! English! , when you go for a job, you need English, you should speak fluent English.

Rasel: Are you comfortable with your bilingual identity?

Ibrahim: Yes. But sometimes different words confuse me.

\section{Interview 2}

Interviewer:Rasel, Participant: Redwan

Rasel: Are you interested in speaking more than one language and which languages?

Redwan: Yes

Rasel: Which languages?

Redwan: Arabic,Bengali,quite a few different languages...because if I go to another country , I don't know their languages..because I can't communicate...

Rasel: Why do you use more than one language?

Redwan: Because, sometimes people talk English with me...but what happens...if I go to Hong kong , ..Something bad if I don't understand their language

Rasel: Which language do you usually speak at home and why?

Redwan: I speak Bengali and English...my dad and grandparents , they speak Bengali and they brought up from Bangladesh...

Rasel: Do you use your heritage language in the place where you live? When and in which situations?

Redwan: I do sometimes...quite a few places, if it is necessary I don't use it...when I come home, I use it with my dad and grandparents

Rasel: If you were given the opportunity, which language would you want to be included in the school curriculum apart from English?

Redwan: Yea, there is a few languages I want to know: Arabic, Bengali. I will choose Arabic, because the Holy Quran is written in Arabic, so that's why I wanna learn Arabic. 
Rasel: What do you think students learn Standard English at schools?

Redwan: It's good to know Standard English....people might not understand poor English

\section{Interview 3}

Interviewer: Rasel, Participant: Sania

Rasel: Are you interested in speaking more than one language and which languages?

Sania: Yea...Italian, Bengali and English

Rasel: Why do you use more than one language?

Sania: I born in Italy and it is my native language, I need more than one language for better Communication.

Rasel: Which language do you usually speak at home and why?

Sania: I speak Italian at home. It is native language. My parents are fluent in Italian.

Rasel: Do you use your heritage language in the place where you live? When and in which situations?

Sania: I don't ...people don't understand Italian

Rasel: If you were given the opportunity, which language would you want to be included in the school curriculum apart from English?

Sania: Definitely Italian, I feel comfortable with Italian Language,

Rasel: What do you think students learn Standard English in school?

Sania: I need it for my education. I can get a good future with good English

\section{Interview 4}

Interviewer:Rasel, Participant: Hisham

Rasel: Are you interested in speaking more than one language and which languages?

Hisham: Yea...Bengali and English

Rasel: Why do you use more than one language?

Hisham: I use English for my school, they don't get my heritage language. I use Bengali for my home.

Rasel: Which language do you usually speak at home and why?

Hisham: As you know, my parents does not understand good English. I must speak Bengali with them.

Rasel: Do you use your heritage language in the place where you live? When and in which situations?

Hisham: Yea...I use it quite often.I have many friends who speak Bengali.I do social chat and do fun

Rasel: If you were given the opportunity, which language would you want to be included in the school curriculum apart from English?

Hisham: Bengali, we can learn heritage culture more when it is included in school curriculum Rasel: What do you think students learn Standard English in school?

Hisham: I want to study in a good university and I need good English for that.

\section{Pedagogical approaches and suggestions}

Research in the teaching of heritage languages is "limited" (Anderson, 2008, p. 83). However, recent development in the theoretical pedagogical approaches has suggested the communicative language teaching (CLT) approach to teach heritage languages (Anderson, 2008, Kenner \& Ruby, 2012). Recent development of the CLT has focused more on the role of grammar in the language classroom. Because of this development, some researchers refer CLT to post-communicative approach (Pachler, 2000 in Anderson, 2008, p. 84). The researcher would like to concentrate on post-CLT in teaching ethnic minority languages. 
I would like to discuss communicative approach in brief before providing suggestions in the light of findings. The development of CLT dates back to the 1960s. Its aims have become much broader and the means of achieving these aims have become clearer with a more authentic focus on the role of grammar (Anderson, 2008). The main secret of CLT is "the communicative competence" (Canale, 1983). CLT focuses on the four language skills: Reading, Writing, Listening and speaking in real life situations. It investigates the functional, communicative and structural aspects of languages. Broadly, CLT emphasizes meaning in communication in real life situations through using it. It also applies translations and whatever things are needful for the effective and appropriate use of languages. CLT has developed strong connections with the socio cultural contexts. It enables language learners authentically to when, where, how and to whom use the target language with communicative competence. In order to show a more advanced insight of CLT, Canale and Swain (1980 in Canale, 1983) developed four dimensions of communicative competence: grammatical competence, sociolinguistic competence, Discourse competence and strategic competence. Briefly, the communicative language teaching approach is much more learner-oriented (Anderson, 2008: 86).

I identified a number of useful suggestions to teach heritage languages in the light of literature review and analysis of questionnaires and interviews. I believe that brainstorming, directed activities related to texts, collaborative project work (Anderson, 2008), use of social web, parental support, storytelling by grandparents (Kenner \& Ruby, 2012) can contribute much more to the cognitive development of the multilingual learners. Moreover, activities like role play, pair work, group work and drama are worthwhile in developing literacy practices of the ethnic minorities in London.

\section{Scaffolding and Social interaction}

Coyle, Hood \& Mash (2010, p. 29) underpin the notion that more capable children or adults should provide scaffolding for children's endeavors. Children should be given support when needed. The adult should modify the amount of help that a child is given in scaffolding mode. Nevertheless, an adult person should intervene to prevent the child regressing (Bruner, 1978), while over the time, the child will become less reliant on others to perform the task correctly. During the presentation of Montessori materials, the teacher always modifies how much help the child is given. It depends on the teacher's close observations and knowledge of the child. In this regard, Rogoff attempts to modify Bruner's deficiencies while building on Vygotsky's ZPD. However, Rogoff criticizes scaffolding and ZPD for the weighing of control towards the adult; she affirms that the child's cognitive development is embedded in the context of social relationships and sociocultural tools. Accordingly, Rogoff (1990) developed the concept of guided participation; which is a form of an apprenticeship. It is based on Vygotsky's social construction where the knowledge is socially constructed and a product of culture. This idea involves the collaborative process which happens between children and adults. 
Guided participation is a very effective way to teach early years children. The following activity can clarify this process.

Teacher/Capable peer: Teacher cuts a piece of paper in the shape of a Bengali letter [न] which represents the "N" sound in English. He shows it to his students.

Child: The child looks at the shape and tries to make one by himself. He asks the teacher the name of the Bengali letter.

Teacher: The teacher explains $[$ ब] $=\mathrm{N}$. The teacher shows other Bengali letters which are similar in meaning and pronunciation.

Child: The child is aware of the two different letters with similar meaning and pronunciation.

It eases his learning of heritage language alphabets.

Teacher: Teacher shows other Bengali letters

Child: He tries repeatedly and gets ideas of Bengali letters.

This conversation shows that teacher's or more capable peer's assistance in teaching heritage language can benefit early year's children. Once memorization is completed, children's competence in Bengali language alphabets becomes stronger. After this competence, ideas of Bengali word formation can be shown. For example,

$\begin{array}{ll}\text { Bengali } & \text { English } \\ \text { বাবা }(\text { Baba })= & \text { Father } \\ \text { মা }(\mathrm{ma})= & \text { Mother } \\ \text { বোন }(\text { Bon })= & \text { Sister } \\ \text { ভাই }(\text { Bhai }= & \text { Brother } \\ \text { বন্ধু (Bondhu) }= & \text { Friend }\end{array}$

Teachers can ask students to bring some family photos. Later, teachers ask the students about the people in the picture. Teachers explain the relationships in the picture in Bengali for example. These students are aware of words which show relationship. Most of them use this heritage language words like grandmother নোনী, father বাবা) at home. Now they are aware of their use of words in the heritage language. Their concept of heritage language words start developing. Once they have good command of word formation, teachers take initiatives to start teaching sentence formation in heritage languages which shows authentic context and helps students to achieve communicative competence.

The following example shows how teachers can teach heritage language with the help of English sentence formation:

\section{Sentence formation in Bengali and English}

\begin{tabular}{|l|l|l|l|}
\hline $\begin{array}{l}\text { Subject+Verb+Object } \\
\text { (English) }\end{array}$ & $\begin{array}{l}\text { Note: we add s/es with } \\
\text { the verb if the subject is } \\
\text { like"He/She/it/Peter" }\end{array}$ & $\begin{array}{l}\text { कर्তा(S)+कर्म(O)+ } \\
\text { क्रिয়া(V) } \\
\text { (বাংলा)Bengali }\end{array}$ & $\begin{array}{l}\text { Note: the verbs in } \\
\text { Bengali do not take } \\
\text { s/es like English }\end{array}$ \\
\hline I eat rice. & & $\begin{array}{l}\text { आমि ভाত খাই (I rice } \\
\text { eat) The meaning in } \\
\text { Bengali is logical. } \\
\text { However, it shows } \\
\text { illogical sentence } \\
\text { formation in English }\end{array}$ & \\
\hline
\end{tabular}


Teachers can teach these differences very easily. Teachers can first write the subjects of both languages and then verbs and then objects on white board and explain orally the meaning of each sentence in Bengali and English. Students will be aware of the differences of the two languages.

The analysis of word and sentence formation enables students gain some competence in both heritage and the English language. The next step is to involve students in many communicative activities through storytelling. Popular stories told by children's grandparents should be collected for children's bilingual development.

The following are the examples of two Bengali popular folk stories:

The Fox and the Crocodile- a folk story from Bangladesh

"A long time ago, near a small village by a shimmering lake, in the shadow of the green mountains, there lived a clever fox and his faithful friend, the crocodile..." Full story can be retrieved from: http://www3.hants.gov.uk/story-2.pdf .

\section{Buri and the Marrow in English \& Bengali-a folk story from Bangladesh}

"An old woman travels through the forest to meet her daughter. On her way she meets a fox, a tiger and a lion - and they all want to eat her! Buri tells them to wait until she returns, when she'll be nice and fat. When it is time to go home, her daughter thinks of a plan to outwit the tiger and the lion. But will the fox be fooled? Full story can be retrieved from http://www.languagelizard.com/Buri-and-theMarrow-p/buri.htm .

\section{Teaching Language through Literature}

Popular traditional folk stories are good source of literature. Most of the children in early year's schools are familiar with the traditional folk stories. The previous paragraphs showed word and sentence formations of Bengali language. Children can be involved in pair work, group work and brainstorming through traditional literature reading.

\section{Transliteration and translation}

Traditional folk stories can be transliterated in heritage languages. It will develop children's existing knowledge. Children's knowledge with word and sentence structure can be enhanced by transliteration system. For example the folk story "Burry and the Marrow" can be transliterated and translated into Bengali. Below is the shining example of this:

“এক বুরি তার মেয়ের সাথে দেখা কর্যার জন্য বনের ভিতর দি|য় পার হচ্ছিল পথে তার বাঘ,শিয়াল আর শিংহে সাথে দেখা হল সবাই তাকে খেতে চাłল বুরি তাদের অপেক্ষা করতে বলল যাতে সে মটা তাড়া হয়ে আসতে পারে |ফেরার সময় হল তার মে একটি পরিকল্পনা আটল যাতে সবাই বোকা হয় কিন্ত শিয়ালকে কি বোকা বানানো সম্ভব ?.."

\section{Transliteration}

ek buri tar mer sathe dakha korar jonno bonar vitor dia jassilo. Pothe se akti bug, shingho abong sial delklo jara takhe khete chailo. Buri bollo se tar mer bari theke khei mota hoye asbe. Tokhon tara kheye moja pabe...... 
Children can be asked to read the English version of the story first. After that they can read the transliteration and finally read the translation in Bengali. It is also possible to show in other languages. After getting the clear idea of the story, they become ready for different communicative activities like matching words, fill in gaps, answering to questions and multiple choices.

\section{Pair work}

Teachers can make pairs of the students of his class and ask them to talk to his peer about the story. It is a five minutes activity. Students participate in pair work and try to find out the Bengali meaning of the story.

\section{Word matching}

After that, the teacher asks all the students to come to the white board one by one and write the Bengali or the English word they discussed and learned.

\section{For example,}

পত্নী (Old woman)
তার (?)
মেয়ে (Daughter)
(?) Forest
মা (Mother)
শিয়াল (?)
বাঘ (Tiger)
সিংহের (Lion)

\section{Writing}

After that, the teacher can ask the students to write answers to some questions. For example,

Where was the Buri (old woman) going? (বুরি কোথায় যাচ্ছে?) (buri kothai jasse)

What was the problem on her way? (তোর পথে সমস্যা কি ছিল?) (ter pothe ki somasha hoyechilo)

How did she survive? (সে কিভাবে বেঁচে ছিল?) (se ki vabe bechechilo)

Students can be given fifteen minutes to write answers to these questions. The teacher can silently monitor their progress. Finally the teacher checks spelling and grammar. In this section, there is special emphasis on Bengali and English grammar in an authentic context.

\section{Grammar in authentic context}

\section{স্কুলের পরে, আমি বাড়িতে যাব}

Skul er pore ami bari jabo

After school I will go home.

During the class, the teacher explains the use of future tense in Bengali and English. For example, in the above sentence, "will" in the English sentence and "ব" in the Bengali sentence indicate future. The teacher also explains that there is no 
adding of s/es with verbs in Bengali. Therefore, students can use all verbs with all subjects. Students become aware of future tense and third person singular number. Later, the teacher can ask the students to bring ideas of another popular folk story and describe it in the next class with proper use of tense and number. Finally, the teacher asks students to describe their daily routine following tense and number in their heritage languages. The teacher monitors their mistakes and corrects them.

\section{Parental support in Bilingual learning}

Parental support is very important in developing a child's bilingual mind. Daily conversation is very effective. It helps in habit formation. Parents should keep contact with their children's respective school and follow up children's bilingual development. They should speak in a standard form of heritage language at home. This will develop children's bilingual mind. Parents can select interesting heritage stories and tell their children in their free time. However, it may raise issues for parents who cannot speak both standard heritage language and the English language. In this situation, social media can be a good solution.

\section{Social media in teaching heritage language}

Social media is very important in teaching and learning languages. There is a wide range of social media on the internet. However, popular social media are Facebook, Google Plus, YouTube, Twitter, Linked In, and institutional social blog. Social media challenges traditional models of literacy and allows people to communicate and collaborate. It also gives people an audience and removes hierarchy as it is built from the bottom up. Social media is open and transparent.

Schools in ethnic minority areas can activate some types of social media like blogging to enhance learning and teaching. It allows less capable learners to communicate with more capable peers and teachers. It creates a social bond. Social web browsers can write their own problem of learning. They can sometimes use their heritage language in transliteration in their school blog and get feedback in the same language. Ethnic minority children can communicate and find friends with the same language community. Children are engaged and more fascinated by popular social media like "Facebook". They can create ideas for using Facebook. The structured interview and questionnaires I have used in this study show that most of the children use Facebook and communicate with their heritage language community. Facebook has a translation system. They can use their heritage language, English or transliteration on the Facebook wall and keep in touch with their friends and family. Therefore, the use of social media for whole school communication needs to be included within the whole school communications policy. Collaborations between schools and parents can enable children to upload family pictures on social media and write comments in their heritage languages.

\section{Inclusion of heritage language in the national curriculum}

Inclusion of heritage languages in the National Curriculum of England for primary schools would greatly benefit ethnic minority children. This would allow 
mainstream schools to engage ethnic minority children in communicative heritage language activities. This would also promote children's confidence that their heritage languages are respected in mainstream schools and they will feel that they are a part of the wider society. This would also increase the opportunity to use and develop their knowledge and skills in their first languages. Professionals in bilingualism have come "to recognize the importance of first language development for children learning English as an additional language (NALDIC, 2009, p. 1)". Adopting a bilingual pedagogical approach in early years would subscribe to the view that a bilingual pedagogy is beneficial to all children and young people, those who are bilingual and monolingual and those who are learning EAL. Many bilingual staff in mainstream schools should provide valuable bilingual support to learners, helping them and their parents access the curriculum and the routines and procedures of the schools in early years setting (NALDIC, 2009, p. 2).

\section{Conclusion}

The study attempted to find an acceptable approach to teaching multilingualism in a primary school context in East London. A number of suggestions have been underpinned to break the silence of bilingual children (Kenner \& Ruby, 2012). In addition, the need for collaborations between mainstream school and complementary schools in East London has been emphasized. This study has suggested suitable approaches to teaching heritage languages to ethnic minority school children in multilingual contexts and data was collected through questionnaires, interviews, and a literature review. It also focused on the role of policy makers forming a bilingual school curriculum which could create room for the bilingual children. It showed how heritage languages can be helpful in developing the target language. The study also showed how learning reaches a successful level when it is scaffolded onto existing knowledge (Hall \& Cook, 2012).

Bilingual children can therefore show their identity in the wider society through respect and recognition. Inclusion of a bilingual syllabus in the early years would be helpful as most of the acquisition starts from childhood (Krashen, 1982). Mainstream school teachers should sometimes visit complementary schools to see the progress and situation of ethnic minority children (Kenner \& Ruby, 2012). This would increase the level of confidence of the ethnic minority children to use their heritage languages in mainstream schools. A bilingual curriculum would probably help prevent school bullying of ethnic minority children.

It is very important that different approaches and methods should be used to teach ethnic minority languages. I considered a post communicative approach to teach ethnic minority languages. Activities like pair work, group work, role play, brainstorming, multiple choice and filling in the gaps have been suggested in this study. Moreover, parental support has been considered very important in developing children's bilingual mind. Social media like Facebook and School Blog have been emphasized. Children feel curious and interested in online learning. Therefore, learning can occur in a number of ways like blogging on the school social web, personal information exchanging on a Facebook wall, transliteration on a 
social web, writing heritage language in Facebook and Twitter. This would promote bilingual learning. Storytelling from grandparents can be a good source of fun and entertainment for bilingual children. It would enhance bilingual learning to a great extent. Finally, a convenient environment of bilingual learning could be established through the inclusion of a bilingual curriculum in early years. Bilingual children should also be aware of the competition of the wider society and prepare themselves to be competent participants in the job market and business.

\section{Acknowledgements}

I am deeply indebted to the editors of the Working Papers in Literacy, Culture, and Language Education for guidance and instructions and the two anonymous reviewers for their invaluable suggestions that really helped me to refine this paper. I would be responsible for all remaining inaccuracies.

\section{References}

Anderson, J., \& Obied, V. (2011). Languages, literacies and learning: from monocultural intercultural perspectives. NALDIC Quarterly, 8(3).

Anderson, J. (2008).Towards an integrated second-language pedagogy for foreign and community/heritage languages in multilingual Britain. Language Learning Journal, 36(1), 79-89.

Baker, C. (2011). Foundations of bilingual education and bilingualism (5th Ed.). USA: McNaughton and Gunn Ltd.

Barkow, H (2013). Buri and the Marrow in English \& Bengali-a folk story from Bangladesh Source. Retrieved from http://www.languagelizard.com/Buriand-the-Marrow-p/buri.htm

Bourdieu, P. (1991). Language and symbolic power, Cambridge, Polity Press.

Bruner, J. S. (1978). The process of Education, London: Harvard University press.

Canale, M. (1983). From Communicative competence to communicative language pedagogy. In Language and communication, ed. J. Richards and R. Schmidt. Harlow: Longman.

Chomsky, N. (2007). Approaching UG from below. Interfaces+recursion=language? Chomsky's minimalism and the view from syntax-semantics. Mouton: Berlin.

Coyle, D., Hood, P., \& Mash, D. (2010). Content and language integrated learning.

Cambridge: CUP.

Cummins, J. (1984). Bilingualism and special education: Issues in assessment and pedagogy. GB: Short Run Press Ltd.

Cummins, J. (1981). Bilingualism and minority-language children. Ontario: Ontario Institute for Studies in Education.

Hall, G., \& Cook, G. (2012). Own-language use in language teaching and learning.In Language Teaching, 45:3, 271-308. 
Harmer, J. (2003). The practice of English language teaching. London: Longman.

Hampshire Bilingual Learners Support Service (BLSS). (2002). The fox and the crocodile- a folk story from Bangladesh Source. Retrieved from http://www3.hants.gov.uk/story-2.pdf

Kenner, C., \& Ruby, M, (2012). Interconnecting Words. Stoke on Trent: Trentham Books Ltd.

Krashen, S. (1982). Principles and Practice in Second Language Acquisition. Oxford: Pergamon.

Miller, P. H. (2010). Theories of Developmental Psychology (5th Ed.), USA: Worth Publishers.

NALDIC (2009). Working paper 9: Developing a bilingual pedagogy for UK schools.

Pennycook, A. (1994). The cultural politics of English as an international language. London: Longman.

Phillipson, R. (1992). Linguistic imperialism. UK: Oxford University Press.

Rogoff, B. (1990). Apprenticeship in thinking: Cognitive Development in Social Context. UK: Oxford University Press.

Said, E. (1978). Orientalism. USA: Vintage Books.

Smith, P. K. (1997). Bullying in life-span perspective: What can studies of school bullying and workplace bullying learn from each other? Journal of Community \& Applied Social Psychology, 7, 249-255.

Vygotsky, L. S. (1978). Mind in society. Cambridge, MA: Harvard University Press. 


\section{Appendix}

\section{Questionnaire on Multilingualism}

1. How many languages do you speak?
A. 1
B. 2
C. 3 D. 4
E. 5 or more

2. Apart from English what other languages do you speak?
A. Bengali
B. Hindi
C. Urdu
D. specify.....

3. Do you speak this language in your daytime school?
A. Sometimes
B. Not at all

If the answer is B, could you tell me why do you not speak the heritage language in daytime school?

4. Do you think your heritage language is related to your identity?
A. Yes
B. No Give a reason.........

5. Do you speak your heritage language at home?
A. Not at all
B. Sometimes
C. Always

6. Why don't you use English at home?
A. My parents can't speak English
B. My grandparents cannot speak English
C. Not applicable

7. Do you learn your heritage language in a weekend complementary school?
A. Yes
B. No
C. Used to

8. Why do you learn this language?
A. To retain my bilingual identity
B. To communicate with my grandparents
C. Not applicable

9. Do you think your heritage language should be valued in your daytime school?
A. Definitely
B. Sometimes
C. No. Give reasons.....

10. Do you think knowing your heritage language can be used to teach different subjects?
A. No
B. $30 \%$
C. $40 \%$
D. Specify....

11. Do you think heritage language can help you improve your English?
A. Yes
B. No
C. Give a reason.....

12. Do you think there should be a teaching assistant in your heritage language in your classroom?
A. Yes
B. No
C. Give a reason........

13. Do you think your heritage language should be included in your school curriculum?
A. Yes
B. No
C. Give a reason.....

14. If you were given the opportunity, would you be interested in showing that you know more than one language?
A. Yes
B. No
C. Give a reason..... 
15. If you do not attend a complementary school, how do you learn your heritage language?

A. Story telling by grandparents B. Daily conversation with parents C. Internet and media D. Other.....

16. Do you see any problem with using your heritage language in the place where you live?
A. No
B. Yes
C. Give reasons.....

17. Are you afraid of speaking your heritage language in your school?
A. Yes
B. No
C. Give reasons.....

18. Do you think you must learn Standard English with a Standard English accent?

19. Yes $\quad$ B. No C. Give reasons......

20. Why do you have to learn Standard English at school?
A. Better future
B. to get a place in a good university C. Specify.....

21 Do you think you can contribute more to the place where you live by learning your heritage language?
A. Yes
B. No
C. Give reasons........

\section{Author Note}

Mohammad Rasel Howlader is a PhD student in Education (TESOL) at the University of the West of Scotland. He holds three Master's degrees: MA in English from the University of Rajshahi, MA in Applied Linguistics from the University of Bedfordshire, UK, and MA in Education from Goldsmiths, University of London. Rasel's research interest includes but not limited to TESOL, Applied linguistics and Bilingual education.

Correspondence concerning this article should be addressed to Mohammad Rasel Howlader, PhD student, School of Education, Paisley Campus, PA1 2BE, Scotland, UK.

Contact: Mohammad-Rasel.Howlader@uws.ac.uk 